volume follows others in the series in its light modernization of spelling and punctuation and inclusion of a glossary of unfamiliar words, both of which enhance its accessibility and help to bring it into conversation with its modern audience. Demers has produced a very fine and full edition of the Bacon/Jewel Apology or Answer in Defence of the Church of England that should be invaluable to scholars and graduate students working in early modern women's writing, Elizabethan history, and the history and theology of the English Reformation.

PATRICIA BRACE

Laurentian University

Barbier-Mueller, Jean-Paul.

Dictionnaire des poètes français de la seconde moitié du XVI siècle (15491615). A-B.

Avec la collaboration de Nicolas Ducimetière et la participation de Marine Molins.

Travaux d'Humanisme et Renaissance, 540. Genève : Librairie Droz, 2015. 1000 p. ISBN 978-2-600-0851-7 (relié) $79 €$.

Barbier-Mueller, Jean-Paul.

Dictionnaire des poètes français de la seconde moitié du XVI siècle (15491615). C-D.

Avec la collaboration de Nicolas Ducimetière et la participation de Marine Molins.

Travaux d'Humanisme et Renaissance, 544. Genève : Librairie Droz, 2015. 967 p. ISBN 978-2-600-0852-4 (relié) $79 €$.

Barbier-Mueller, Jean-Paul.

Dictionnaire des poètes français de la seconde moitié du XVI siècle (15491615). E-J.

Avec la collaboration de Nicolas Ducimetière et la participation de Marine Molins.

Travaux d'Humanisme et Renaissance, 544. Genève : Librairie Droz, 2016. 748 p. ISBN 978-2-600-01905-7 (relié) $79 €$.

Complétant la publication toujours en cours de son catalogue intitulé $M a$ collection poétique consacré aux poètes de langue française du $\mathrm{XVI}^{\mathrm{e}}$ siècle 
(1973-2005), les trois premiers volumes du Dictionnaire des poètes français de la seconde moitié du XVI siècle de Jean-Paul Barbier-Mueller se présentent comme une somme importante pour tous les chercheurs et autres « curieux » qui veulent s'informer sur la poésie française de la Renaissance. Au fil des décennies, le collectionneur genevois a en effet conservé, en plus des exemplaires rarissimes de recueils poétiques, de très précieuses notices sur les différents protagonistes de l'effervescente vie littéraire française. Chacune des notices donne le plus grand nombre d'informations possibles sur la carrière des poètes, en citant les éditions connues, en insérant des renseignements biographiques, mais aussi les jugements des contemporains, ainsi que certaines analyses empruntées à la littérature critique. En reprenant, tout en les corrigeant, les analyses anciennes et les recensements de Goujet (Bibliothèque françoise, 1745), Picot (Les Français italianisants au XVI siècle, 1907) ou Arbour (L'Ére baroque, 1977-1980), le travail de Jean-Paul Barbier-Mueller s'avère être extrêmement précieux comme bilan biobibliographique. Il fait ainsi le point sur les connaissances actuelles de la vie poétique française, en se consacrant essentiellement aux règnes d'Henri II et d'Henri III : la date terminale, 1615, renvoie à la mort de la « reine Margot », même si les raisons de la présence ou de l'absence de certaines figures (Claude Hopil par exemple) ne sont pas toujours très claires.

Écrit avec un style personnel, nerveux et fleuri, cet inventaire a l'avantage d'approfondir, du moins pour les écrits poétiques, certaines notices du Dictionnaire des lettres françaises $d u$ XVI siècle de Grente, qui avaient été revues et augmentées par l'équipe dirigée par Michel Simonin (2001). Comme dans ce précédent Dictionnaire, les minores sont ici bien représentés (de Nicolas Bonyer, Pierre Cheminart à Guillaume du Sable), au même titre que les grands noms du panthéon des lettres de la Renaissance française (Du Bellay, Desportes, Baiff, d'Aubigné...). On s'en doute, c'est bien plus pour la première catégorie, ces auteurs oubliés qui ont parfois peu produit et sur lesquels la bibliographie est mince, que ce Dictionnaire est le plus utile. Le lecteur peut ainsi découvrir quels furent les choix de carrière des poètes français et les resituer dans le panorama général de la vie littéraire. L’importance donnée aux réseaux de sociabilité, aux mécènes et aux protecteurs, à la place des épîtres dédicatoires et aux poèmes liminaires, mais aussi aux recueils collectifs, aide à s'orienter dans cet immense corpus : les différentes étapes d'une carrière, de la recommandation initiale à l'écriture parallèle dans d'autres champs du savoir (le droit par exemple), sont régulièrement soulignées et font voir les différentes possibilités qui s'offraient à 
celui ou celle qui voulait se faire un nom dans les lettres. Le rapport entre Paris et la province n'est pas encore celui d'un départ unilatéral vers la métropole culturelle : la trajectoire d'un poète comme Pey de Garros, qui publia uniquement des vers en gascon, rappelle la diversité des lettrés français face au centralisme émergent. Entre les poètes qui n'ont publié qu'un seul recueil, comme Pierre de Cornu ou Christofle du Pré-Passy, et les "polygraphes inépuisables », comme Philibert Bugnyon ou François Du Souhait dont les noms sont présents sur une foule d'imprimés, l'écart est considérable et le Dictionnaire de Barbier-Mueller réussit à restituer très concrètement la richesse et la diversité de la vie poétique dans la France de la seconde moitié du XVI e siècle.

Sur les grands noms du panthéon poétique, les notices sont aussi utiles, mais elles peuvent aussi se révéler parfois plus décevantes pour l'interprétation, voire pour la mise au point bibliographique - celle sur Du Bellay, notamment, pose problème -, mais ce n'est sans doute pas l'objectif de l'ouvrage. Il est cependant dommage que certaines éditions savantes n’aient pas été consultées, comme par exemple la récente édition des Euvres du sieur de Fiefmelin ou celles de la Défense de Du Bellay, de même que certains volumes d'études critiques, comme celui sur François Habert, également ignorés. On retrouvera dans ce Dictionnaire la place essentielle occupée par l'imitation des poètes italiens, ainsi que l'influence de Ronsard (de Claude Binet à Marc-Claude de Buttet), mais ce qui constitue à nos yeux l'intérêt de ce travail repose sur deux autres apports.

Tout d'abord, la hiérarchisation entre les différents poètes : la place accordée aux minores ne saurait occulter le fait que tous ne se valent pas et, même si l'auteur s'emballe parfois (par exemple pour dénoncer la «médiocrité » d'un Guillaume Du Buys), il est aussi nécessaire de signifier dans cet immense inventaire que toutes les plumes ne sont pas d'une égale importance - même si certaines méritent en effet de sortir des oubliettes de l'histoire littéraire. Ensuite, ce Dictionnaire redonne à la poésie militante et à l'inscription des poètes dans les guerres de religion tout leur poids, en indiquant la complexité de leurs trajectoires religieuses et politiques, des conversions aux revirements, sans pour autant figer les frontières confessionnelles : si un Jean Fornier passe de la foi catholique à la Réforme, d'autres poètes réformés modérés ne tournent pas le dos à un public catholique, comme Du Bartas ou encore Gilbert Gondouyn, qui soumet son recueil à la censure des docteurs en théologie de la Sorbonne. 
Enfin, les trois premiers volumes de ce Dictionnaire poétique rappellent le volume impressionnant des vers pamphlétaires ou partisans produits dans la seconde partie du siècle : c'est bien l'imbrication passionnante des poètes français dans les combats de leur temps qui est retracée dans ces différentes notices. Extrêmement bien documenté et agréablement écrit, ce Dictionnaire des poètes français de la seconde moitié du XVI siècle est bien un outil de recherche essentiel pour qui s'intéresse à la poésie française de la Renaissance.

GRÉGOIRE HOLTZ

University of Toronto (Victoria College)

\section{Beecher, Donald.}

Adapted Brains and Imaginary Worlds: Cognitive Science and the Literature of the Renaissance.

Montreal and Kingston: McGill-Queen's University Press, 2016. Pp. ix, 484. ISBN 978-0-7735-4681-3 (paperback) \$39.95.

The potential benefits of applying cognitive science to an understanding of the arts are twofold: one may gain an understanding of the workings of human cognition that illuminates artistic processes and content; from this understanding may come suggestions for prescriptive and proscriptive approaches that increase the likelihood of artistic success. The pitfalls are also twofold: the first, arising from the second noted benefit, is the imposition of rigid notions that hinder creative freedom and lead to a narrow recognition of artistic success, notions, moreover, that are dictated to the arts from the outside; the second, arising from a lack of real cross-disciplinary expertise, is a situation in which accounts of findings in cognitive science and their applicability to artistic activity are presented by those who don't really have the understanding or judgment to back up their claims to an audience not in a position to evaluate them.

Work on cognitive science and the arts has been flourishing for some years now. I am most familiar with it in the area of theatre studies. Donald Beecher's Adapted Brains and Imaginary Worlds brings cognitive science and its "hermeneutic usefulness" (45) to the understanding of some literary works of the Renaissance, mostly English. Beecher's book, I argue, offers the benefits and pitfalls listed above. 